\title{
Diffraction by a Cylindrical Obstacle
}

Charles H. Papas

Citation: Journal of Applied Physics 21, 318 (1950); doi: 10.1063/1.1699662

View online: http://dx.doi.org/10.1063/1.1699662

View Table of Contents: http://aip.scitation.org/toc/jap/21/4

Published by the American Institute of Physics

\section{Articles you may be interested in}

The Diffraction Produced by Cylindrical and Cubical Obstacles and by Circular and Square Plates The Journal of the Acoustical Society of America 10, 6 (2005); 10.1121/1.1915950

Absorption and Scattering for Impedance Boundary Conditions on Spheres and Circular Cylinders The Journal of the Acoustical Society of America 20, 108 (2005); 10.1121/1.1906352

Directionality Patterns for Acoustic Radiation from a Source on a Rigid Cylinder

The Journal of the Acoustical Society of America 24, 46 (2005); 10.1121/1.1906846

Incomplete Atomic Arrangement in Crystals

The Journal of Chemical Physics 3, 592 (2004); 10.1063/1.1749733

Self-repair of ordered pattern of nanometer dimensions based on self-compensation properties of anodic porous alumina

Applied Physics Letters 78, 826 (2001); 10.1063/1.1344575

Electric Dipoles in the Presence of Elliptic and Circular Cylinders

Journal of Applied Physics 22, 14 (2004); 10.1063/1.1699813

\section{A.P| Journal of Applied Physics}

Save your money for your research.

It's now FREE to publish with us -

no page, color or publication charges apply.

Publish your research in the Journal of Applied Physics

to claim your place in applied physics history. 


\title{
Diffraction by a Cylindrical Obstacle*
}

\author{
Charles H. Papas \\ Cruft Laboratory, Harvard University, Cambridge, Massachusetts
}

(Received September 23, 1949)

The diffraction of a plane electromagnetic wave by an infinitely long, perfectly conducting cylinder has been treated by a variational method (see the two papers by H. Levine and J. Schwinger). The incident field is assumed to be polarized in the direction of the cylinder axis, and thus the entire field is of two-dimensional nature. This formulation yields an expression for the diffracted cylindrical wave amplitude, at distances from the cylinder large compared to its transverse dimension and the wave-length, which is stationary relative to small independent variations of the surface currents arising from plane-wave excitation along a pair of directions in space; furthermore, the stationary form of the diffracted amplitude is independent of the scale of the surface currents. In accordance with a theorem of Levine and Schwinger, the total plane-wave scattering cross section is simply related to the diffracted cylindrical wave amplitude in the direction of incidence. To examine the high frequency behavior of the cross section, the surface current induced by a plane wave is taken different from zero only on the illuminated part of the cylinder, where its value is derived from the tangential component of the incident magnetic field. The resulting cross section is obtained and is shown to approach $4 a$ when $k a$ approaches infinity ( $k=2 \pi \div$ wave-length, $a$ equals the radius of cylinder).

\section{INTRODUCTION}

$T$ HE theoretical examination of the diffraction of a steady-state plane electromagnetic wave by a cylindrical obstacle has interested many investigators. One of the earliest treatments proceeds according to the Fourier-Lamé eigenfunction method. The incident electromagnetic wave is assumed to be polarized parallel to the axis of the perfectly conducting, circular cylinder. The axis of the cylinder lies along the $x$ axis, and the incident wave has only an $x$ component of the electric field, $E_{x}{ }^{\text {inc }}(\rho, \phi)$, and a $y$ component of the magnetic field, $H_{y}{ }^{\text {inc }}(\rho, \phi)$ (Fig. 1). For an incident wave whose electric field is of unit amplitude and propagating in the direction $\phi_{1}$, i.e., $E_{x}{ }^{\text {inc }}=\exp \left[i k \rho \cos \left(\phi-\phi_{1}\right)\right]$, the corresponding scattered electric field is given by

$$
\begin{aligned}
E_{x}{ }^{s c}(\rho, \phi & \left.-\phi_{1}\right) \\
& =-\sum_{-\infty}^{\infty}(i)^{m} \frac{J_{m}(k a)}{H_{m}{ }^{(1)}(k a)} H_{m}{ }^{(1)}(k \rho) \cos m\left(\phi-\phi_{1}\right),
\end{aligned}
$$

where $J_{m}(x)$ are the Bessel functions, and $H_{m}{ }^{(1)}(x)$ are the Hankel functions of the first kind, and $k=2 \pi$ $\div$ wave-length.
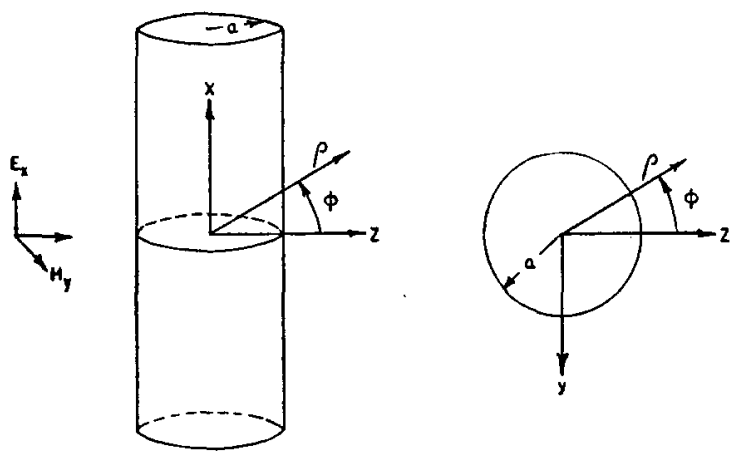

FIG. 1. Diffracting circular cylinder

${ }^{*}$ The research reported in this document was made possible through support extended Cruft Laboratory, Harvard University, jointly by the Navy Department (ONR), the Signal Corps of the U. S. Army, and the U. S. Air Force, under ONR Contract N5ori76, T. O. I.
The inadequacy of the rigorous representation (1) stems from the fact that its convergence becomes slower with increasing values of $k a$, i.e., at high frequencies. To avoid this difficulty, Debye ${ }^{1}$ represented the scattered field by a Fourier integral in the following manner. Let

$$
f(m)=\frac{H_{m}^{(1)}\left(k_{\rho}\right)}{H_{m}{ }^{(1)}(k a)} \cos m\left(\phi-\phi_{1}\right),
$$

where $m$ can be considered to be a continuous real variable. According to the Fourier integral theorem,

$$
\begin{aligned}
& f(m)=\frac{1}{2 \pi} \int_{-\infty}^{\infty} d \mu \int_{-\infty}^{\infty} f(\alpha) \exp [i \mu(m-\alpha)] d \alpha \\
&=-\frac{1}{\pi} \int_{-\infty}^{\infty} d \mu \exp (i \mu m) \int_{0}^{\infty} f(\alpha) \cdot \cos \mu \alpha \cdot d \alpha \\
&=\frac{1}{\pi} \int_{-\infty}^{\infty} d \mu \exp (i \mu m) \int_{0}^{\infty} \frac{H_{\alpha}^{(1)}(k \rho)}{H_{\alpha}^{(1)}(k a)} \\
& \cdot \cos \alpha\left(\phi-\phi_{1}\right) \cdot \cos \mu \alpha \cdot d \alpha .
\end{aligned}
$$

Then,

$$
\begin{aligned}
& E_{x}{ }_{x} c\left(\rho, \phi-\phi_{1}\right)=--\frac{1}{\pi} \sum_{m=-\infty}^{\infty}(i)^{m} J_{m}(k a) \\
& \int_{-\infty}^{\infty} d \mu \exp (i \mu m) \int_{0}^{\infty} \frac{H_{\alpha}^{(1)}(k \rho)}{H_{\alpha}{ }^{(1)}(k a)} \cos \alpha\left(\phi-\phi_{1}\right) \cdot \cos \mu \alpha \cdot d \alpha .
\end{aligned}
$$

Since

$$
\sum_{m=-\infty}^{\infty}(i)^{m} J_{m}(k a) \exp (i m \mu)=\exp (i k a \cos \mu),
$$

by interchanging the order of summation and integra-

${ }^{1} \mathrm{P}$. Debye, "Das electromagnetische Feld um einem Zylinder und die Theorie des Regenbogens," Physik. Zeits. 9, 775-778 (1908). 
tion Debye's integral representation is obtained:

$$
\begin{aligned}
& E_{x}{ }^{8 c}\left(\rho, \phi-\phi_{1}\right)=-\frac{1}{\pi} \int_{-\infty}^{\infty} \exp (i k a \cos \mu) d \mu \\
& \times \int_{0}^{\infty} \frac{H_{\alpha}^{(1)}(k \rho)}{H_{\alpha}{ }^{(1)}(k a)} \cos \alpha\left(\phi-\phi_{1}\right) \cdot \cos \mu \alpha \cdot d \alpha .
\end{aligned}
$$

By an approximate evaluation of (2) for large $k a$, he obtained

$E_{x}{ }_{x}^{c c}(\rho, \phi)=-\left(\frac{k a \cos (\phi / 2)}{2 k \rho}\right) \exp \left[i k\left(\rho-2 a \cos \frac{\phi}{2}\right)\right]$

for the scattered electric field resulting from a plane wave incident in the direction $\phi_{1}=0$.

Up to the present we have had the rigorous eigenfunction expansion of the scattered field (1) which suffers from convergence difficulties for large $k a$, and the Debye summation scheme yielding the expression (3) for the high frequency far-zone scattered field, valid for directions not in the neighborhood of $\phi=\pi$. Consequently (3) cannot be used to calculate the total scattering cross section of the cylinder; (1) can be used, however, even for large values of $k a$, i.e., at high frequencies, but only with considerable heuristic manipulation of the slowly convergent eigenfunction expansion.

It is the purpose of this paper to show that the aforementioned difficulties can be avoided if the problem is formulated from the start using a variational principle in the manner of Levine and Schwinger., ${ }^{3}$ This formulation yields an expression for the diffracted cylindrical wave amplitude, at distances from the cylinder large compared to its transverse dimension and the wavelength, which is stationary relative to small independent variations of the surface currents arising from plane-wave excitation along a pair of directions in space. Furthermore, the stationary form of the diffracted amplitude is independent of the scale of the surface currents. The total plane-wave scattering cross section is simply related to the diffracted cylindrical wave amplitude in the direction of incidence. To examine the high frequency behavior of the cross section $(k a \gg 1)$, the surface current induced by a plane wave is taken different from zero only on the illuminated part of the cylinder, where its value is derived from the tengential component of the incident magnetic field.

\section{INTEGRAL EQUATION FORMULATION}

Consider a plane electromagnetic wave incident upon a perfectly conducting circular cylinder of radius $a$, whose axis lies along the $x$ axis of a rectangular coordinate system, Fig. 1 . The incident wave is polarized parallel to the axis of this cylinder, its electric field

\footnotetext{
${ }^{2} \mathrm{H}$. Levine and J. Schwinger, "On the theory of diffraction by an aperture in an infinite plane screen, I," Phys. Rev. 74, 958-974 (1948).

${ }^{3} \mathrm{H}$. Levine and J. Schwinger, "On the theory of diffraction by an aperture in an infinite plane screen, II," Phys. Rev. 75, 1423$1432(1949)$.
}

FIG. 2. Integration domain.

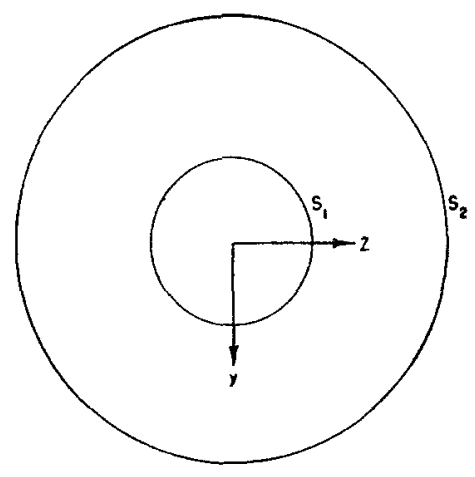

being given by

$$
E_{x}^{\text {inc }}(\rho, \phi)=\exp \left[i k_{\rho} \cos \left(\phi-\phi_{1}\right)\right],
$$

where $\rho, \phi$ are cylindrical coordinates, $k=2 \pi \div$ wavelength, and $\phi_{1}$ is the direction of propagation. The scattered electric field, $E_{x}^{s c}(\rho, \phi)$, is also polarized parallel to the cylinder axis. The electric field at any point $r, r$ being a two-dimensional vector from the origin to any point $(\rho, \phi)$, is the sum of the incident and scattered fields, $E_{x}(r)=E_{x}{ }^{\text {inc }}(r)+E_{x}{ }^{s c}(r)$. The problem is formulated in terms of these electric fields. To emphasize their two-dimensional scalar nature, denote them by $\psi(r) \equiv E_{x}(r), \quad \psi^{\text {inc }}(r) \equiv E_{x}{ }^{\text {inc }}(r), \quad$ and $\psi^{s c}(r)$ $\equiv E_{x}{ }^{80}(r)$.

Note that $\psi(r)$ satisfies the scalar wave equation

$$
\left(\nabla^{2}+k^{2}\right) \psi(r)=0,
$$

and the two-dimensional scalar Green's function $G\left(r, r^{\prime}\right)$ satisfies the inhomogeneous scalar wave equation

$$
\left(\nabla^{2}+k^{2}\right) G\left(r, r^{\prime}\right)=-\delta\left(r-r^{\prime}\right),
$$

where the inhomogeneous term is the two-dimensional Dirac delta-function. Applying Green's second scalar identity to $\psi(r)$ and $G\left(r, r^{\prime}\right)$, we obtain

$$
\begin{array}{r}
\int_{v}\left[G\left(r^{\prime}, r\right)\left(\nabla^{\prime 2}+k^{2}\right) \psi\left(r^{\prime}\right)-\psi\left(r^{\prime}\right)\left(\nabla^{\prime 2}+k^{2}\right) G\left(r^{\prime}, r\right)\right] d \tau \\
=\int_{s_{1}+s_{2}}\left[G\left(r^{\prime}, r\right) \frac{\partial}{\partial n^{\prime}} \psi\left(r^{\prime}\right)-\psi\left(r^{\prime}\right) \frac{\partial}{\partial n^{\prime}} G\left(r^{\prime}, r\right)\right] d s^{\prime},
\end{array}
$$

where the derivative in the surface integral is taken in the direction of the outward normal (Fig. 2). The surface integral extends over the surface of the scattering cylinder, $s_{1}$, and a concentric cylinder, $s_{2}$, of very large radius. In view of (2) and (3) the volume integral becomes $\psi(r)$, and since $\psi(r)=0$ when $r$ lies on the scattering cylinder, it follows that

$$
\begin{aligned}
& \psi(r)=\int_{s_{1}} G\left(r^{\prime}, r\right) \frac{\partial}{\partial n^{\prime}} \psi\left(r^{\prime}\right) d s^{\prime} \\
& \quad+\int_{s_{2}}\left[G\left(r^{\prime}, r\right) \frac{\partial}{\partial n^{\prime}} \psi\left(r^{\prime}\right)-\psi\left(r^{\prime}\right) \frac{\partial}{\partial n^{\prime}} G\left(r^{\prime}, r\right)\right] d s^{\prime} .
\end{aligned}
$$

Recalling that $\psi(r)=\psi^{\text {inc }}(r)+\psi^{s c}(r)$, the integral over $s_{2}$ 
in (5) becomes

$$
\begin{aligned}
\int_{s_{2}}\left[G\left(r^{\prime}, r\right)\right. & \left.\frac{\partial}{\partial n^{\prime}} \psi^{\text {inc }}\left(r^{\prime}\right)-\psi^{\text {inc }}\left(r^{\prime}\right) \frac{\partial}{\partial n^{\prime}} G\left(r^{\prime}, r\right)\right] d s^{\prime} \\
& +\int_{s_{2}}\left[G\left(r^{\prime}, r\right) \frac{\partial}{\partial n^{\prime}} \psi^{s c}\left(r^{\prime}\right)-\psi^{s c}\left(r^{\prime}\right) \frac{\partial}{\partial n^{\prime}} G\left(r^{\prime}, r\right)\right] d s^{\prime} .
\end{aligned}
$$

The first integral yields $\psi^{\text {ine }}(r)$ and the second disappears due to the asymptotic behavior of the integrand for $r^{\prime} \rightarrow \infty$, and (5) takes the form

$$
\psi(r)=\psi^{\mathrm{inc}}(r)+\int_{s_{1}} G\left(r^{\prime}, r\right) \frac{\partial}{\partial n^{\prime}} \psi\left(r^{\prime}\right) d s^{\prime} .
$$

Since $\left(\partial / \partial n^{\prime}\right) \psi\left(r^{\prime}\right)=-\left(\partial / \partial \rho^{\prime}\right) E_{x}\left(\rho^{\prime}, \phi^{\prime}\right)=i \omega \mu H_{\phi}\left(\rho^{\prime}, \phi^{\prime}\right)$ and $d s^{\prime}=a d \phi^{\prime},(6)$ becomes

$$
\begin{aligned}
& \psi(\rho, \phi)=\psi^{\mathrm{inc}}(\rho, \phi) \\
& +\int_{0}^{2 \pi} i \omega \mu H_{\phi}\left(a, \phi^{\prime}\right) G\left(a, \phi^{\prime} ; \rho, \phi\right) \cdot a d \phi^{\prime}
\end{aligned}
$$

$H_{\phi}\left(a, \phi^{\prime}\right)$ is numerically equal to the surface-current density at the point $\left(a, \phi^{\prime}\right)$ on the cylinder. Let $I_{\phi_{1}}\left(\phi^{\prime}\right)$ $=i \omega \mu a H_{\phi}\left(a, \phi^{\prime}\right)$, where $I_{\phi_{1}}\left(\phi^{\prime}\right)$ is proportional to the current density at the point $\left(a, \phi^{\prime}\right)$ due to the incident wave (1) propagating in the direction $\phi_{1}$. With this notation, (7) becomes

$$
\psi(\rho, \phi)=\psi^{\mathrm{inc}}(\rho, \phi)+\int_{0}^{2 \pi} I_{\phi_{1}}\left(\phi^{\prime}\right) G\left(a,{\phi^{\prime}}^{\prime} ; \rho, \phi\right) d \phi^{\prime} .
$$

Since $\psi(a, \phi)=0$, i.e., $E_{x}$ must disappear at the surface of the cylinder, the following integral equation is obtained by placing $\rho=a$ in (8):

$$
0=\psi^{\mathrm{inc}}(\rho, \phi)+\int_{0}^{2 \pi} I_{\phi_{1}}\left(\phi^{\prime}\right) G\left(a, \phi^{\prime} ; a, \phi\right) d \phi^{\prime}
$$

or

$$
-\exp \left[i k a \cos \left(\phi-\phi_{1}\right)\right]=\int_{0}^{2 \pi} I_{\phi_{1}}\left(\phi^{\prime}\right) G\left(a, \phi^{\prime} ; a, \phi\right) d \phi^{\prime} .
$$

The solution of this integral equation would yield $I_{\phi_{1}}(\phi)$, i.e., the current except for a numerical factor, produced by a plane incident wave traveling in the direction $\phi_{1}$. By substituting this solution back into (8) and carrying out the indicated integration, the diffracted field $\psi(\rho, \phi)$ could be determined. Two objections are immediately apparent, the first is the difficult problem of solving (9) for $I_{\phi_{1}}\left(\phi^{\prime}\right)$, the second is the evaluation of the integral in (8) once $I_{\phi_{1}}\left(\phi^{\prime}\right)$ is determined. Moreover, to obtain the total scattering cross section, still another integration would have to be performed. Use will be made of the integral equation (9) to formulate a variational principle for the scattered wave amplitude.

\section{VARIATIONAL PRINCIPLE FOR SCATTERED WAVE AMPLITUDE}

Since the two-dimensional Green's function, $G\left(r, r^{\prime}\right)$, has to satisfy the inhomogeneous wave equation (3) and the radiation condition, i.e., it must represent at large distances from the cylinder outwardly propagating cylindrical waves, it follows that $G\left(r, r^{\prime}\right)=(i / 4) H_{0}$ $\times\left(k\left|r-r^{\prime}\right|\right)$. For large $r$, the leading term of the asymptotic expansion is

$r \rightarrow \infty: G\left(r, r^{\prime}\right)$

where

$$
\sim \frac{i}{4}\left(\frac{2}{\pi k\left|r-r^{\prime}\right|}\right)^{\frac{1}{2}} \exp (i k)\left|r-r^{\prime}\right| \exp \left(-i-\frac{\pi}{4}\right),
$$

$\left|r-r^{\prime}\right|=\left[\rho^{2}+\rho^{\prime 2}-2 \rho \rho^{\prime} \cos \left(\phi-\phi^{\prime}\right)\right]$

$$
\sim \rho\left(1-\frac{\rho^{\prime}}{\rho} \cos \left(\phi-\phi^{\prime}\right)\right) \text {. }
$$

With these asymptotic values the scattered field, which is given by the integral in (8), at large distances becomes

$$
\begin{aligned}
\psi^{s c}(\rho, \phi) \sim & \frac{i}{4}\left(\frac{2}{\pi i k \rho}\right)^{\frac{1}{2}} \exp (i k \rho) \\
& \times\left[\int_{0}^{2 \pi} \exp \left[-i k a \cos \left(\phi-\phi^{\prime}\right)\right] I_{\phi_{1}}\left(\phi^{\prime}\right) d \phi^{\prime}\right] .
\end{aligned}
$$

The scattered wave amplitude is represented by the quantity in square brackets. We denote it by $A\left(\phi, \phi_{1}\right)$ :

$$
A\left(\phi, \phi_{1}\right)=\int_{0}^{2 \pi} \exp \left[-i k a \cos \left(\phi-\phi^{\prime}\right)\right] I_{\phi_{1}}\left(\phi^{\prime}\right) d \phi^{\prime} .
$$

As the notation explicitly denotes, $A\left(\phi, \phi_{1}\right)$ is the scattered wave amplitude in the direction $\phi$ for an incident wave traveling in the direction $\phi_{1}$.

Multiplication of the integral equation (9) by $I_{\phi_{2}}(\phi)$ and integration with respect to $\phi$ (in accordance with the notation that $I_{\phi_{2}}(\phi)$ is $i \omega \mu a$ times the surfacecurrent density at the point $(a, \phi)$ due to an incident plane wave traveling in the direction $\phi_{2}$ ) leads to

$$
\begin{aligned}
-\int_{0}^{2 \pi} \exp \left[i k a \cos \left(\phi-\phi_{1}\right)\right] I_{\phi_{2}}(\phi) d \phi \\
\quad=\int_{0}^{2 \pi} \int_{0}^{2 \pi} d \phi I_{\phi_{2}}(\phi) G\left(a, \phi^{\prime} ; a, \phi\right) I_{\phi_{1}}\left(\phi^{\prime}\right) d \phi^{\prime} .
\end{aligned}
$$

But according to (10),

$$
\begin{array}{r}
\int_{0}^{2 \pi} \exp \left[i k a \cos \left(\phi-\phi_{1}\right)\right] I_{\phi_{2}}(\phi) d \phi \\
=\int_{0}^{2 \pi} \exp \left[-i k a \cos \left(\pi+\phi_{1}-\phi\right)\right] I_{\phi_{2}}(\phi) d \phi \\
=A\left(\pi+\phi_{1}, \phi_{2}\right) .
\end{array}
$$


$A\left(\pi+\phi_{1}, \phi_{2}\right)$ represents the scattered wave amplitude in the direction of $\pi+\phi_{1}$ due to an incident wave traveling in the direction $\phi_{2}$, and it is clear that it must equal the scattered wave amplitude in the direction $\pi+\phi_{2}$ due to an incident wave traveling in the direction $\phi_{1}$. This reciprocity relation is given by the equality,

$$
A\left(\pi+\phi_{1}, \phi_{2}\right)=A\left(\pi+\phi_{2}, \phi_{1}\right) .
$$

Dividing (11) by

$$
\begin{aligned}
\int_{0}^{2 \pi} \exp [i k a \cos (\phi & \left.\left.-\phi_{1}\right)\right] I_{\phi_{2}}(\phi) d \phi \\
& \times \int_{0}^{2 \pi} \exp \left[i k a \cos \left(\phi-\phi_{2}\right)\right] I_{\phi_{1}}(\phi) d \phi,
\end{aligned}
$$

and taking into consideration (12) and (13),

$$
\frac{1}{1\left(\pi+\phi_{2}, \phi_{1}\right)}=\frac{1}{A\left(\pi+\phi_{1}, \phi_{2}\right)}=-\frac{\int_{0}^{2 \pi} \int_{0}^{2 \pi} d \phi I_{\phi_{2}}(\phi) G\left(a, \phi^{\prime} ; a, \phi\right) I_{\phi_{1}}\left(\phi^{\prime}\right) d \phi^{\prime}}{\int_{0}^{2 \pi} \exp \left[i k a \cos \left(\phi-\phi_{1}\right)\right] I_{\phi_{2}}(\phi) d \phi \int_{0}^{2 \pi} \exp \left[i k a \cos \left(\phi-\phi_{2}\right)\right] I_{\phi_{1}}(\phi) d \phi} .
$$

The expression (14) is homogeneous in $I_{\phi_{1}}(\phi), I_{\phi_{2}}(\phi)$ and stationary with respect to independent first-order variations about their correct values (determined by integral equations of the form (9)). The property of homogeneity is clear. To show the stationary property of (14), proceed as follows. Clearing fractions in (14),

$$
\begin{gathered}
\int_{0}^{2 \pi} \exp \left[i k a \cos \left(\phi-\phi_{1}\right)\right] I_{\phi_{2}}(\phi) d \phi \\
\times \int_{0}^{2 \pi} \exp \left[i k a \cos \left(\phi-\phi_{2}\right)\right] I_{\phi_{1}}(\phi) d \phi \\
=-A \int_{0}^{2 \pi} \int_{0}^{2 \pi} d \phi I_{\phi_{2}}(\phi) G\left(\phi, \phi^{\prime}\right) I_{\phi_{1}}\left(\phi^{\prime}\right) d \phi^{\prime},
\end{gathered}
$$

where $A \equiv A\left(\pi+\phi_{1}, \phi_{2}\right), G\left(\phi^{\prime}, \phi\right) \equiv G\left(a, \phi^{\prime} ; a, \phi\right)$. Now take the variations of $I_{\phi_{2}}(\phi), I_{\phi_{1}}(\phi)$, and $A$.

$$
\begin{aligned}
& \int_{0}^{2 \pi} \exp \left[i k a \cos \left(\phi-\phi_{1}\right)\right] \delta I_{\phi_{2}}(\phi) d \phi \\
& \quad \times \int_{0}^{2 \pi} \exp \left[i k a \cos \left(\phi-\phi_{2}\right)\right] I_{\phi_{1}}(\phi) d \phi \\
& +\int_{0}^{2 \pi} \exp \left[i k a \cos \left(\phi-\phi_{1}\right)\right] I_{\phi_{2}}(\phi) d \phi \\
& \times \int_{0}^{2 \pi} \exp \left[i k a \cos \left(\phi-\phi_{2}\right)\right] \delta I_{\phi_{1}}(\phi) d \phi_{1} \\
& =-\delta A \int_{0}^{2 \pi} \int_{0}^{2 \pi} d \phi I_{\phi_{2}}(\phi) G\left(\phi, \phi^{\prime}\right) I_{\phi_{1}}\left(\phi^{\prime}\right) d \phi^{\prime} \\
& -A \int_{0}^{2 \pi} \int^{2 \pi} d \phi \delta I_{\phi_{2}}(\phi) G\left(\phi, \phi^{\prime}\right) I_{\phi_{1}}\left(\phi^{\prime}\right) d \phi^{\prime} \\
& -A \int_{0}^{2 \pi} \int_{0}^{2 \pi} d \phi I_{\phi_{2}}(\phi) G\left(\phi, \phi^{\prime}\right) \delta I_{\phi_{1}}\left(\phi^{\prime}\right) d \phi^{\prime} .
\end{aligned}
$$

Using (12) and (13) this can be written in the form

$$
\begin{aligned}
& A \int_{0}^{2 \pi} \exp \left[i k a \cos \left(\phi-\phi_{1}\right)\right] \delta I_{\phi_{2}}(\phi) d \phi \\
& +A \int_{0}^{2 \pi} \exp \left[i k a \cos \left(\phi-\phi_{2}\right)\right] \delta I_{\phi_{1}}(\phi) d \phi \\
& =-\delta A \int_{0}^{2 \pi} \int_{0}^{2 \pi} d \phi I_{\phi_{2}}(\phi) G\left(\phi, \phi^{\prime}\right) I_{\phi_{1}}\left(\phi^{\prime}\right) d \phi^{\prime} \\
& -A \int_{0}^{2 \pi} \int_{0}^{2 \pi} d \phi \delta I_{\phi_{2}}(\phi) G\left(\phi, \phi^{\prime}\right) I_{\phi_{1}}\left(\phi^{\prime}\right) d \phi^{\prime} \\
& -A \int_{0}^{2 \pi} \int_{0}^{2 \pi} d \phi I_{\phi_{2}}(\phi) G\left(\phi, \phi^{\prime}\right) \delta I_{\phi_{1}}\left(\phi^{\prime}\right) d \phi^{\prime} .
\end{aligned}
$$

Combining terms, this equation becomes

$$
\begin{aligned}
& \delta A \int_{0}^{2 \pi} \int_{0}^{2 \pi} d \phi I_{\phi_{2}}(\phi) G\left(\phi, \phi^{\prime}\right) I_{\phi_{1}}\left(\phi^{\prime}\right) d \phi^{\prime} \\
& =-A \int_{0}^{2 \pi} d \phi \delta I_{\phi_{2}}(\phi)\left[\int_{0}^{2 \pi} d \phi^{\prime} G\left(\phi, \phi^{\prime}\right) I_{\phi_{1}}\left(\phi^{\prime}\right)\right. \\
& \left.+\exp \left[i k a \cos \left(\phi-\phi_{1}\right)\right]\right]-A \int_{0}^{2 \pi} d \phi^{\prime} \delta I_{\phi_{1}}\left(\phi^{\prime}\right) \\
& {\left[\int_{0}^{2 \pi} d \phi G\left(\phi, \phi^{\prime}\right) I_{\phi_{2}}(\phi)\right.} \\
& \left.+\exp \left[i k a \cos \left(\phi^{\prime}-\phi_{2}\right)\right]\right] .
\end{aligned}
$$

If $I_{\phi_{1}}(\phi)$ and $I_{\phi_{2}}(\phi)$ satisfy integral equations of the form (9), then the square-bracket terms in (15) vanish identically. Hence $A$ is stationary with respect to firstorder independent variations of $I_{\phi_{1}}(\phi)$ and $I_{\phi_{2}}(\phi)$.

\section{A THEOREM CONCERNING THE TOTAL SCATTERING CROSS SECTION AND SCATTERED WAVE AMPLITUDE}

The total scattering cross section is defined by the ratio of the total scattered power per unit length of 
cylinder to the incident power per unit area. The timeaverage incident power per unit area, $\bar{S}^{\text {inc }}$, is given by $\frac{1}{2} \operatorname{Re} E \times H^{*}=\frac{1}{2} \operatorname{Re} E^{*} \times H$, where the asterisk indicates the conjugate complex and Re the real part. Since

and

$$
E_{x}^{\text {inc }}=\exp (i k z)
$$

then

$$
H_{y}^{\mathrm{inc}}=\frac{1}{i \omega \mu}-\frac{\partial}{\partial z} E_{x}^{\mathrm{ine}}=\frac{k}{\omega \mu} \exp (i k z),
$$

$$
\bar{S}^{\text {inc }}=\frac{1}{2} \operatorname{Re} E_{x}{ }^{{ }^{i n c}} H_{y}{ }^{\text {inc }}=\frac{k}{2 \omega \mu} .
$$

The time-average scattered power, $\bar{P}^{s c}$, passing through a cylinder $s_{2}$ of large radius is equal to the time-average power leaving the cylindrical surface $s_{1}$, i.e.,

$$
\begin{aligned}
\bar{P}^{s c} & =\operatorname{Re} \int_{s_{2}} \frac{1}{2}\left(E_{x}^{s c}(\rho, \phi)\right)\left(-H_{\phi}^{s c}(\rho, \phi)\right) \rho d \phi \\
& =\operatorname{Re} \int_{s_{1}} \frac{1}{2}\left(E_{x}^{s c}(a, \phi)\right)\left(-H_{\phi}^{s c}(a, \phi)\right) a d \phi .
\end{aligned}
$$

The total scattering cross section, $\sigma$, is then given by the expression

$$
\sigma=\frac{\bar{P}^{s c}}{\bar{S}^{\text {inc }}}=\frac{1}{k}-\operatorname{Re}_{i}^{1} \int_{0}^{2 \pi} E_{x}{ }^{* c c}(a, \phi) \frac{\partial}{\partial \rho} E_{x}{ }^{s c}(a, \phi) a d \phi .
$$

At the surface of the cylinder, $E_{x}{ }^{* s c}(a, \phi)=-E_{x}{ }^{* \text { inc }}(a, \phi)$; hence

$\sigma=\frac{1}{k} \operatorname{Re} \frac{1}{i} \int_{0}^{2 \pi}-E_{x} *^{i n e}(a, \phi) \frac{\partial}{\partial \rho}-\left[E_{x}(a, \phi)\right.$

But $\left.-E_{x}^{\text {inc }}(a, \phi)\right] a d \phi$.

$$
\int_{0}^{2 \pi} E_{x}{ }^{i \mathrm{inc}}(a, \phi) \frac{\partial}{\partial \rho} E_{x}^{\mathrm{inc}}(a, \phi) a d \phi=0
$$

and consequently

$$
\sigma=\frac{1}{k} \operatorname{Re}_{i}^{1} \int_{0}^{2 \pi} \exp \left[-i k a \cos \left(\phi-\phi_{1}\right)\right] I_{\phi_{1}}(\phi) d \phi,
$$

where use has been made of $a(\partial / \partial \rho) E_{x}(a, \phi)=-i_{\omega \mu} a H_{\phi}$ $\times(a, \phi)=-I_{\phi_{1}}(\phi)$. In view of $(10)$ and (17) $\sigma$ can be represented in terms of the scattered wave amplitude:

$$
\sigma=\frac{1}{k} \operatorname{Re}_{i}^{1} A\left(\phi_{1}, \phi_{1}\right)=\frac{1}{k} \operatorname{Im} A\left(\phi_{1}, \phi_{1}\right)
$$

where Im means imaginary part.

\section{LOW FREQUENCY SCATTERING CROSS SECTION}

To find the total scattering cross section it is necessary first to compute $A\left(\phi_{1}, \phi_{1}\right)$ from (14) and then to use (18). Let $\phi_{1}=0$ and $\phi_{2}=\pi$ in (14); then

$$
\frac{1}{A(\pi, \pi)}=\frac{\int_{0}^{2 \pi} \int_{0}^{2 \pi} d \phi I_{\pi}(\phi) G\left(a, \phi^{\prime} ; a, \phi\right) I_{0}\left(\phi^{\prime}\right) d \phi^{\prime}}{\int_{0}^{2 \pi} \exp (i k a \cos \phi) I_{\pi}(\phi) d \phi \int_{0}^{2 \pi} \exp (-i k a \cos \phi) I_{0}(\phi) d \phi} .
$$

At low frequencies $k a \ll 1$; also choose $I_{\pi}(\phi)=I_{0}(\phi)=1$. The numerator then becomes

$$
\begin{array}{r}
\int_{0}^{2 \pi} \int_{0}^{2 \pi} d \phi G\left(a, \phi^{\prime} ; a, \phi\right) d \phi^{\prime}=-\frac{i}{4} \int_{0}^{2 \pi} \int_{0}^{2 \pi} d \phi d \phi^{\prime} H_{0}\left(k\left|r-r^{\prime}\right|\right) \\
=\frac{i}{4} \int_{0}^{2 \pi} \int_{0}^{2 \pi} d \phi d \phi^{\prime} \sum_{m=0}^{\infty} \epsilon_{m} J_{m}(k a) H_{m}^{(1)}(k a) \cos m\left(\phi-\phi^{\prime}\right) \\
=i \pi^{2} J_{0}(k a) H_{0}(k a) .
\end{array}
$$$$
\text { and }
$$

$$
\begin{aligned}
\int_{0}^{2 \pi} \exp (i k a \cos \phi) d \phi & =2 \pi J_{0}(k a), \\
& \int_{0}^{2 \pi} \exp (-i k a \cos \phi) d \phi=2 \pi J_{0}(k a) .
\end{aligned}
$$

Consequently

and

$$
\frac{1}{A(\pi, \pi)}=-\frac{i \pi^{2} J_{0}(k a) H_{0}(k a)}{\left[2 \pi J_{0}(k a)\right]^{2}}=-\frac{i H_{0}(k a)}{4} \frac{J_{0}(k a)}{,},
$$

Suppose, instead of taking $I_{\pi}(\phi)=I_{0}(\phi)=1$, the following more general representation is used:

$$
\begin{aligned}
I_{0}(\phi)= & \sum_{0}^{\infty} A_{m} \cos m \phi, \\
& I_{\pi}(\phi)=\sum_{0}^{\infty} A_{m} \cos m(\phi-\pi)=\sum_{0}^{\infty} A_{m}(-1)^{m} \cos m \phi,
\end{aligned}
$$

where the $A_{m}$ 's are constants. Substitution in (19) gives for the numerator:

$$
\begin{aligned}
& \int_{0}^{2 \pi} \int_{0}^{2 \pi} d \phi I_{\pi}(\phi) G\left(a, \phi^{\prime} ; a, \phi\right) I_{0}\left(\phi^{\prime}\right) d \phi^{\prime} \\
& \quad=\frac{i}{4} \int_{0}^{2 \pi} \int_{0}^{2 \pi} d \phi d \phi^{\prime}\left(\sum_{m=0}^{\infty} A_{m}(-1)^{m} \cos m \phi\right) \\
& \quad \times\left(\sum_{p=0}^{\infty} \epsilon_{p} J_{p}(k a) H_{p}^{(1)}(k a) \cos p\left(\phi-\phi^{\prime}\right)\right)
\end{aligned}
$$

$$
\times\left(\sum_{n=0}^{\infty} A_{n} \cos n \phi^{\prime}\right) \text {. }
$$


Let

$C_{m n}=(-1)^{m} \int_{4}^{i} \int_{0}^{2 \pi} d \phi d \phi^{\prime} \cos m \phi$

$$
\times\left(\sum_{p=0}^{\infty} \epsilon_{p} J_{p}(k a) H_{p}^{(1)}(k a) \cos p\left(\phi-\phi^{\prime}\right)\right) \cos n \phi^{\prime} .
$$

But

$$
\int_{0}^{2 \pi} \int_{0}^{2 \pi} d \phi d \phi^{\prime} \cos m \phi \cos p\left(\phi-\phi^{\prime}\right) \cos n \phi^{\prime}=\frac{4 \pi^{2} \delta_{p}{ }^{n} \delta_{p}{ }^{m}}{\epsilon_{p}{ }^{2}}
$$

where

$$
\delta_{n}{ }^{m}=\left\{\begin{array}{ll}
1 & m=n \\
0 & m \neq n
\end{array}\right\} \text { and } \epsilon_{m}=\left\{\begin{array}{ll}
1 & m=0 \\
2 & m \geq 1
\end{array}\right\},
$$

and hence

$$
C_{m n}=\frac{(-1)^{m} i \pi^{2}}{\left(\epsilon_{m}\right)} \delta_{n}{ }^{m} J_{m}(k a) H_{m}{ }^{(1)}(k a) .
$$

The numerator becomes the double series,

$$
\sum_{m=0}^{\infty} \sum_{n=0}^{\infty} A_{m} C_{m n} A_{n}
$$

For the denominator the following is obtained:

$$
\begin{aligned}
& \int_{0}^{2 \pi} \exp (i k a \cos \phi) I_{\pi}(\phi) d \phi \int_{0}^{2 \pi} \exp (-i k a \cos \phi) I_{0}(\phi) d \phi \\
& =\int_{0}^{2 \pi} \exp (i k a \cos ) \phi \sum_{m=0}^{\infty} A_{m}(-1)^{m} \cos m \phi d \phi \\
& \times \int_{0}^{2 \pi} \exp (-i k a \cos ) \phi \sum_{n=0}^{\infty} A_{n} \cos n \phi d \phi \\
& =\left(\sum_{m=0}^{\infty} A_{m}(-1)^{m} 2 \pi(i)^{m} J_{m}(k a)\right) \\
& \quad \times\left(\sum_{n=0}^{\infty} A_{n} 2 \pi(-i)^{m} J_{n}(k a)\right) .
\end{aligned}
$$

By setting $B_{m}=2 \pi(-i)^{m} J_{m}(k a)$, it follows that

$$
\frac{1}{A(\pi, \pi)}=\frac{\sum_{m=0}^{\infty} \sum_{n=0}^{\infty} A_{m} C_{m n} A_{n}}{\left(\sum_{l=0}^{\infty} A_{l} B_{l}\right)^{2}} .
$$

In view of the stationary character of $A(\pi, \pi)$, differentiation of (21) with respect to $A_{m}$ gives

$$
A(\pi, \pi) \sum_{n=0}^{\infty} A_{n} C_{m n}=-B_{m} \sum_{n=0}^{\infty} A_{n} B_{n}, m=0,1,2, \cdots
$$

To simplify (22) the coefficients $D_{n}$ are introduced so that

$$
A(\pi, \pi)=-\sum_{n=0}^{\infty} B_{n} D_{n}
$$

where the $D_{n}$ 's are determined by the simultaneous equations

$$
\sum_{n=0}^{\infty} C_{m n} D_{n}=B_{m}, \quad m=0,1,2, \cdots
$$

Since $C_{m n}=0$ when $m \neq n$ only the diagonal terms of (24) are different from zero, i.e., $D_{n}=B_{n} / C_{n n}$, and hence

$$
\begin{array}{r}
A(\pi, \pi)=-\sum_{n=0}^{\infty} \frac{B_{n}{ }^{2}}{C_{n n}}=\sum_{n=0}^{\infty} \frac{\left(\epsilon_{n} 2 \pi(-i)^{n} J_{n}(k a)\right)^{2}}{i \pi^{2}(-1)^{n} J_{n}(k a) H_{n}{ }^{(1)}(k a)} \\
=4 i \sum_{n=0}^{\infty} \epsilon_{n} \frac{J_{n}(k a)}{H_{n}{ }^{(1)}(k a)} .
\end{array}
$$

Applying the theorem (18) to (25) the total scattering cross section is obtained. It is

$$
\sigma=-\frac{4}{k} \sum_{n=0}^{\infty} \frac{\epsilon_{n} J_{n}^{2}(k a)}{J_{n}^{2}(k a)+N_{n}^{2}(k a)},
$$

where use has been made of the form $H_{n}{ }^{(1)}(k a)=J_{n}(k a)$ $+i N_{n}(k a)$. Since $k a \ll 1$, only the first term of (26) contributes appreciably to the value of $\sigma$. Therefore

$$
\sigma=\frac{4}{k} \frac{J_{0}{ }^{2}(k a)}{J_{0}^{2}(k a)+N_{0}^{2}(k a)} .
$$

Comparing (20) with (27) it is seen that the expressions are identical. It appears that for low frequencies $(k a \ll 1)$ very accurate results are obtained by taking the current on the cylinder to be independent of $\phi$.

\section{HIGH FREQUENCY SCATTERING CROSS SECTION}

Now consider the frequency of the incident wave to be high, i.e., $k a \gg 1$. In (14) let $\phi_{1}=3 \pi / 2, \phi_{2}=\pi / 2$, and for this choice the following result is obtained:

$$
\frac{1}{A\left(\pi+\frac{3 \pi \pi}{2}, \frac{-}{2}\right)}=-\frac{\int_{0}^{2 \pi} \int_{0}^{2 \pi} d \phi I_{\pi / 2}(\phi) G\left(a, \phi^{\prime} ; a, \phi\right) I_{3 \pi / 2}\left(\phi^{\prime}\right) d \phi^{\prime}}{\int_{0}^{2 \pi} \exp \left[i k a \cos \left(\phi-\frac{3 \pi}{2}\right)\right] I_{\pi / 2}(\phi) d \phi \int_{0}^{2 \pi} \exp \left[i k a \cos \left(\phi-\frac{\pi}{2}\right)\right] I_{3 \pi / 2}(\phi) d \phi} .
$$


In accordance with the assumed convention, $I_{\pi / 2}(\phi)$ and $I_{3 \pi / 2}(\phi)$ are proportional to the surface currents induced by incident waves propagating in the directions $\pi / 2$ and $3 \pi / 2$ respectively. For an incident wave propagating in the direction $\phi_{1}, H_{\phi}{ }^{\text {inc }}(\rho, \phi)=-(k / \omega \mu)$ $\times \cos \left(\phi-\phi_{1}\right) \exp \left[i k \rho \cos \left(\phi-\phi_{1}\right)\right]$. It is assumed that the high frequency current induced by a plane wave is different from zero only on the illuminated half of the cylinder, where its value is numerically equal to the tangential component of the incident magnetic field. That is, for a trial function we take

$$
I_{\phi_{1}}(\phi)=\text { const. } \cos \left(\phi-\phi_{1}\right) \exp \left[i k a \cos \left(\phi-\phi_{1}\right)\right] \text {. }
$$

Hence,

$$
\begin{aligned}
I_{\pi / 2}(\phi) & =\text { const. } \sin \phi \exp [i k a \sin \phi] & \text { for } \pi \leq \phi \leq 2 \pi \\
& =0 & \text { for } 0 \leq \phi \leq \pi,
\end{aligned}
$$

and

$$
\begin{aligned}
I_{3 \pi / 2}(\phi) & =- \text { const. } \sin \phi \exp [-i k a \sin \phi] & \text { for } 0 \leq \phi \leq \pi \\
& =0 & \text { for } \pi \leq \phi \leq 2 \pi .
\end{aligned}
$$

Substituting these trial functions in (28) and noting that $A(\pi+3 \pi / 2, \pi / 2)=A(\pi / 2, \pi / 2)$, we get

Since

$$
\frac{1}{A\left(\frac{\pi}{2}, \frac{\pi}{2}\right)}=-\frac{\int_{0}^{\pi} d \phi^{\prime} \sin \phi^{\prime} \exp \left[-i k a \sin \phi^{\prime}\right] \int_{\pi}^{2 \pi} \sin \phi \exp (i k a \sin \phi) G\left(a, \phi^{\prime} ; a, \phi\right)}{\int_{\pi}^{2 \pi} \sin \phi d \phi \int_{0}^{\pi} \sin \phi d \phi} .
$$

then

$$
G\left(a, \phi^{\prime} ; a, \phi\right)=\frac{i}{4} H_{0}{ }^{(1)}\left(2 k a\left|\sin \left(\frac{\phi-\phi^{\prime}}{2}\right)\right|\right)
$$

$$
\frac{1}{A\left(\begin{array}{c}
\pi \pi \\
\frac{2}{2} 2
\end{array}\right)}=\frac{i}{16} \int_{0}^{\pi} d \phi^{\prime} \int_{\pi}^{2 \pi} d \phi \sin \phi^{\prime} \sin \phi \exp \left[-i k a\left(\sin \phi^{\prime}-\sin \phi\right)\right] H_{0}^{(1)}\left(2 k a\left|\sin \left(\frac{\phi-\phi^{\prime}}{2}\right)\right|\right) \text {. }
$$

Let $\phi=2 \pi-\xi$. Then $\sin \phi=-\sin \xi, d \phi=-d \xi$, and

$$
\left|\sin \left(\frac{\phi-\phi^{\prime}}{2}\right)\right|=\left|\sin \left(\frac{\xi+\phi^{\prime}}{2}\right)\right|
$$

With this change of variables (30) becomes*

$$
\begin{aligned}
& \frac{1}{A\left(\frac{\pi}{2, \frac{\pi}{2}}\right)}=-\frac{i}{16} \int_{0}^{\pi} d \phi^{\prime} \int_{0}^{\pi} d \xi \sin \phi^{\prime} \sin \xi \\
& \times \exp \left[-i k a\left(\sin \phi^{\prime}+\sin \xi\right)\right] H_{0}{ }^{(1)}\left(2 k a\left|\sin \left(\frac{\xi+\phi^{\prime}}{2}\right)\right|\right) .
\end{aligned}
$$

Let the variables be changed again according to the following transformation

$$
\left(\phi^{\prime}+\xi^{2}\right) / 2=\beta+\pi / 2, \quad\left(\phi^{\prime}-\xi\right) / 2=\alpha .
$$

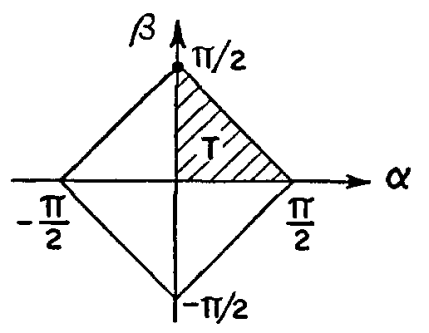

Frg. 3. Integration domain.

* The author received enlightening discussions on integrals of this class from Professor F. Tricomi.
Then

$\sin \phi^{\prime} \sin \xi=\frac{1}{2}(\cos 2 \alpha+\cos 2 \beta), \sin \phi^{\prime}+\sin \xi=2 \cos \alpha \cos \beta$,

$$
\frac{\partial\left(\phi^{\prime}, \xi\right)}{\partial(\alpha, \beta)}=2, \text { and }\left|\sin \left(\left(\xi+\phi^{\prime}\right) / 2\right)\right|=\cos \beta
$$

It follows that in terms of $\alpha$ and $\beta$,

$$
\begin{aligned}
\frac{1}{A\left(\begin{array}{l}
\pi \\
\frac{\pi}{2}-\frac{1}{2}
\end{array}\right)}=-\frac{i}{16} \iint_{Q} \exp (-2 i k a \cos \alpha \cos \beta) \\
\quad \times\left(\cos ^{2} \alpha+\cos 2 \beta\right) H_{0}{ }^{(1)}(2 k a \cos \beta),
\end{aligned}
$$

where $Q$ is the square region in the $\alpha, \beta$ plane shown in Fig. 3. In view of the symmetry in $\alpha$ and $\beta$ about $\alpha=\beta=0$, we can confine the integration to the triangular region $T$, Consequently,

$\frac{1}{A\left(\begin{array}{c}\pi \pi \\ \frac{2}{2} 2\end{array}\right)}=-\frac{i}{4} \int_{0}^{\pi / 2} d \beta \int_{0}^{\pi / 2-\beta} d \alpha \exp (-2 i k a \cos \alpha \cos \beta)$

$$
\times(\cos 2 \alpha+\cos 2 \beta) H_{0}^{(1)}(2 k R \cos \beta) .
$$

Consider first the integration with respect to $\alpha$. Since $k a \gg 1$, the main contribution to the value of this integral occurs in the neighborhood of $\alpha=0$ (provided $\beta \neq \pi / 2$ ). An approximate evaluation is obtained by 
the method of stationary phase:

$$
\begin{aligned}
\int_{0}^{\pi / 2-\beta} d \alpha \exp (-2 i k a \cos \alpha \cos \beta)(\cos 2 \alpha+\cos 2 \beta) \\
=\left(\frac{\pi}{k a}\right)^{\frac{1}{2}} \frac{\cos ^{2} \beta}{(\cos \beta)^{\frac{1}{2}}} \exp (-2 i k a \cos \beta) \exp \left(\begin{array}{c}
\pi \\
i \\
4
\end{array}\right) .
\end{aligned}
$$

There remains the task of evaluating the following integral:

$$
\begin{aligned}
\left.\frac{1}{A\left(\frac{\pi}{2}, \frac{\pi}{2}\right.}\right) & =-\frac{i}{4}\left(\frac{\pi}{k a}\right)^{\frac{1}{2}} \exp \left(\begin{array}{c}
\pi \\
i- \\
4
\end{array}\right) \int_{0}^{\pi / 2} d \beta \frac{\cos ^{2} \beta}{(\cos \beta)^{\frac{1}{2}}} \\
& \times \exp [-2 i k a \cos \beta] H_{0}^{(1)}(2 k a \cos \beta) .
\end{aligned}
$$

As $\beta \rightarrow \pi / 2$ the integrand disappears, and hence the contribution to the integral in the neighborhood of $\beta=\pi / 2$ is very small. When $\beta \neq \pi / 2$ and $k a \rightarrow \infty$,

$H_{0}^{(1)}(2 k a \cos \beta)$

$$
\sim\left(\frac{2}{2 \pi k a \cos \beta}\right)^{\frac{1}{2}} \exp (2 i k a \cos \beta) \exp (-i \pi / 4) .
$$

After substituting this asymptotic form into (33) and changing the upper limit from $\pi / 2$ to $\beta_{0}$, the result is

$$
\frac{1}{A(\pi / 2, \pi / 2)} \sim-\frac{i}{4 k a} \int_{0}^{\beta_{0}} d \beta \cos \beta=-\frac{i}{4 k a} \sin \beta_{0}
$$

for the leading term of the asymptotic development of $[1 / A(\pi / 2, \pi / 2)]$ in reciprocal powers of $k a$. Application of the theorem (18) to (35) gives for the total scattering cross section

$$
\sigma=\frac{1}{k} \operatorname{Im} A\left(\frac{\pi \pi}{2}, \frac{4}{2}\right) \sim \frac{4 a}{\sin \beta_{0}}=\frac{4 a}{\left(1-(4 / k a)^{2}\right)^{\frac{1}{2}}} .
$$

Choose $\beta_{0}$ such that $\cos \beta_{0}=4 / k a$. This choice guarantees the argument of the Hankel function to be equal to or greater than 8 for the entire range of values from $\beta=0$ to $\beta=\beta_{0}$, and thereby allows the replacement of the Hankel function by the first term of its asymptotic

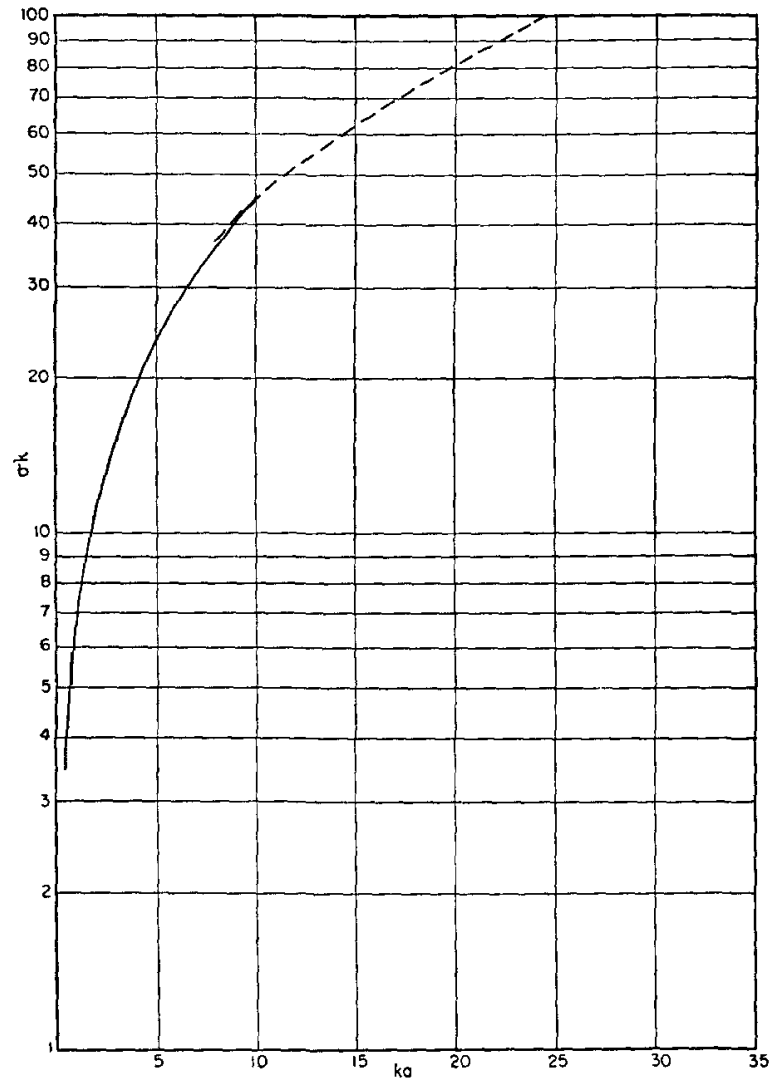

FIG. 4. $\sigma k$ versus ka. Solid curve computed from (26). Broken curve computed from (36).

development. It is seen that as $k a \rightarrow \infty, \sigma \rightarrow 4 a$ which is the geometric optics scattering cross section and physically equal to twice the projected area of a unit length of the scattering cylinder. Figure 4 shows a plot of $\sigma k$ versus $k a$. For values of $k a$ less than 10 Eq. (26) was used to compute $\sigma k$; for $k a$ larger than 8 , Eq. (36) was used. In the region $8<k a<10$ the broken and solid curves overlap.

The author wishes to acknowledge the generous help of Dr. Harold Levine of Harvard University, without whose patient and clear explanations this paper would never have been completed. The assistance of Miss J. Klimas and Mrs. R. Stokey with numerical calculations is gratefully acknowledged. 